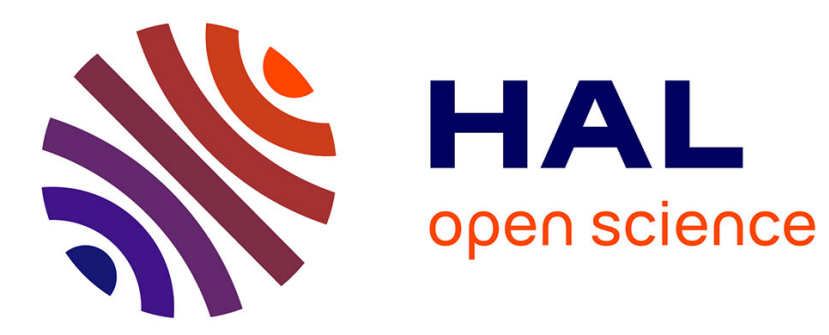

\title{
Stereo reconstruction of semiregular meshes, and multiresolution analysis for automatic detection of dents on surfaces
}

Jean-Luc Peyrot, Frédéric Payan, Natacha Ruchaud, Marc Antonini

\section{- To cite this version:}

Jean-Luc Peyrot, Frédéric Payan, Natacha Ruchaud, Marc Antonini. Stereo reconstruction of semiregular meshes, and multiresolution analysis for automatic detection of dents on surfaces. IEEE International Conference in Image Processing (ICIP), Oct 2014, Paris, France. pp.5417-5421. hal-01032467

\section{HAL Id: hal-01032467 \\ https://hal.science/hal-01032467}

Submitted on 22 Jul 2014

HAL is a multi-disciplinary open access archive for the deposit and dissemination of scientific research documents, whether they are published or not. The documents may come from teaching and research institutions in France or abroad, or from public or private research centers.
L'archive ouverte pluridisciplinaire HAL, est destinée au dépôt et à la diffusion de documents scientifiques de niveau recherche, publiés ou non, émanant des établissements d'enseignement et de recherche français ou étrangers, des laboratoires publics ou privés. 


\title{
STEREO RECONSTRUCTION OF SEMIREGULAR MESHES, AND MULTIRESOLUTION ANALYSIS FOR AUTOMATIC DETECTION OF DENTS ON SURFACES
}

\author{
Jean-Luc Peyrot, Frédéric Payan, Natacha Ruchaud and Marc Antonini, IEEE member \\ Laboratory I3S - University Nice - Sophia Antipolis and CNRS (France) - UMR 7271 \\ peyrot@i3s.unice.fr, fpayan@i3s.unice.fr, ruchaud@i3s.unice.fr, am@i3s.unice.fr
}

\begin{abstract}
Our objective is to include in stereoscopic 3D acquisition systems new technologies to automatically detect deformations on aircraft fuselages. We propose in this paper a semiregular mesh reconstruction dedicated to stereoscopic scanners, combined to a multiresolution analysis tool that detects dents on smooth surfaces. The proposed technique for reconstruction is based on a coarse-to-fine approach, and creates semiregular meshes directly from the stereoscopic images. The output of our scanner is thus a structured mesh, by the way well suited for many applications unlike most of scanners that generate only point clouds. Local distances are then calculated between this semiregular mesh and a smooth version of it, in order to automatically detect dents on the scanned surface. The smooth version is obtained via a technique based on multiresolution analysis. Experimental results show the reliability of our contributions on scanned aircraft fuselages.
\end{abstract}

Index Terms - Semiregular, 3D reconstruction, stereoscopy, acquisition, multiresolution analysis.

\section{INTRODUCTION}

Three main categories of methods are commonly proposed in the literature to acquire and reconstruct surfaces: the systems based on laser time-of-flight [1,2], the systems based on motion techniques [3,4] and systems based on stereovision [5-7]. Many efforts have been done in this category to get efficient and flexible algorithms that finally reconstruct an entire surface from multiple pairs of stereo images.

The context of our current work is the adding of new functionalities in acquisition systems based on stereovision. One of our objectives is to develop a tool that automatically detects local geometric details. According to our partners, we particularly focus on the detection of dents on the fuselage of airplanes.

Multiresolution analysis (MRA) is well suited to detect details in different bands of frequency. This is also available for surfaces, on which geometric deformations can be seen as

THIS WORK IS SUPPORTED BY A GRANT FROM RÉGION PROVENCE ALPES CÔTE D'AZUR variations at different frequencies. When dealing with triangulated surfaces, it is well-known that MRA is more efficient when the mesh is semiregular, i.e. when the mesh has a subdivision connectivity.

As our objective is to include in fine our detection tool in stereoscopic 3D acquisition systems, we propose to first generate a semiregular mesh directly from stereoscopic images (first contribution), before applying a detection algorithm based on multiresolution analysis (second contribution). By the way, we simplify the classical pipeline usually used to get semiregular meshes from scanning systems: point cloud $\rightarrow$ meshing/cleaning $\rightarrow$ semiregular remeshing $\rightarrow$ output semiregular mesh. Several techniques exist to generate semiregular meshes directly from point clouds [8], but to the best of our knowledge, none method directly generates a semiregular mesh from stereoscopic images, despite the interest of such meshes for multiresolution analysis [9], and for many other applications such as compression [10], progressive transmission [11] and so on.

Then, to capture geometric details such as dents, we propose to compare the generated semiregular mesh with a smoother version of it. The latter is obtained by applying MRA (more precisely the butterfly-based lifting scheme) on the semiregular mesh, secondly setting some subbands of wavelet coefficients to zero, and finally applying the synthesis filters. Local distances are then calculated between the two meshes, to detect dents on the scanned surface.

The rest of the paper is organized as follows: Section 2 presents our technique to obtain a semiregular mesh directly from stereoscopic images. Section 3 presents the proposed detection tool based on MRA. Experimental results are given in Section 4, and we finally conclude in Section 5.

\section{SEMIREGULAR MESH RECONSTRUCTION FROM STEREOSCOPIC IMAGES}

\subsection{Overview of the proposed method}

Figure 1(a) illustrates the two main steps of the classical algorithm, used to reconstruct surfaces from stereovision systems like 3D scanners:

1. First, a stereo matching [12] finds pairs of pixels in the 


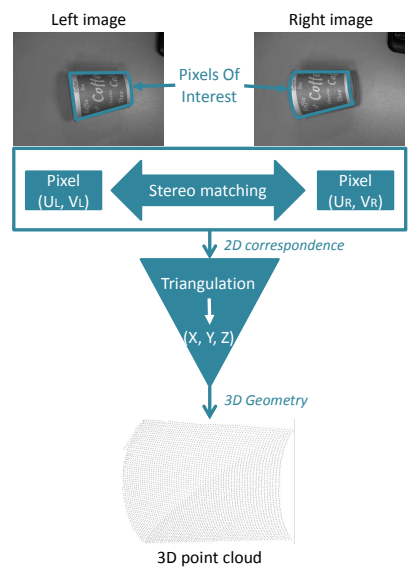

(a) Classical approach.

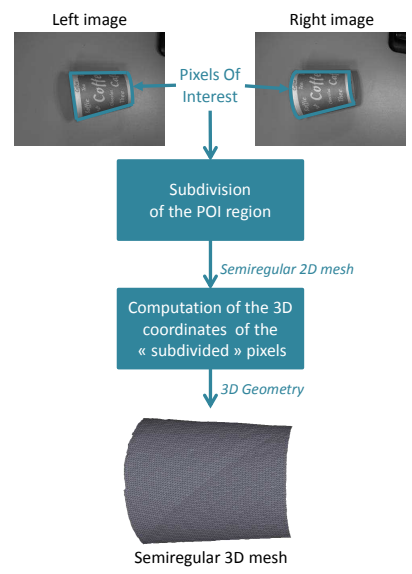

(b) Proposed approach.
Fig. 1. Classical and proposed pipeline to reconstruct surfaces from stereoscopic images.

two stereoscopic images, that represent the same 3D points in the real scene. We call this set of pairs the Pixels Of Interest (POI) region, corresponding to the surrounded blue areas on the top images of Figure 1;

2. Every pair of pixels in the POI region is then used to compute the corresponding 3D coordinates, with a triangulation technique [13].

The output is thus a set of $3 \mathrm{D}$ points, which is often refered in the literature to $3 D$ point cloud. This point cloud is generally dense, since it contains as many 3D points as there are pairs of pixels in the POI region. Moreover, the higher the stereoscopic images resolution, the denser the point cloud will be.

Our approach is quiet different and can be described according to the Figure 1(b) in four steps:

1. Find the POI region in one of the stereoscopic images;

2. Subdivide the POI region on the selected image in order to generate a $2 \mathrm{D}$ semiregular mesh;

3. Compute the $3 \mathrm{D}$ coordinates for each pair of pixels selected by the subdivision (stereo matching + triangulation);

4. Iterate the two last steps, to finally obtain a 3D semiregular mesh of the scanned surface.

Our meshing algorithm has the advantage to only require as inputs one stereoscopic image and the POI region to generate the semiregular connectivity. Moreover, instead of calculating the 3D coordinates for every pixel in the POI region such as done classically (which often leads to an oversampled point cloud/mesh), our approach restrains the computation to the pixels selected by the successive subdivisions, which makes our algorithm faster.
As an example, Figure 2 displays three levels of resolution of the semiregular 3D mesh, generated by our reconstruction method.

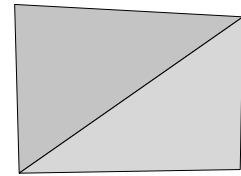

(a)

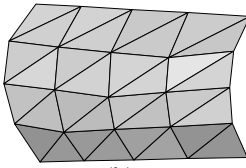

(b)

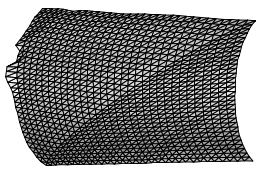

(c)
Fig. 2. Three levels of resolution of a semiregular mesh created with our reconstruction algorithm, and containing (a) 2 triangles; (b) 32 triangles and (c) 2,048 triangles.

\subsection{Details of implementation}

\section{Generation of the 2D semiregular mesh}

As introduced above our reconstruction algorithm first creates a 2D semiregular mesh (in other words a 2D mesh with subdivision connectivity) on the POI region of one stereoscopic image. Such a connectivity will enable a multiscale representation of the corresponding surface. This $2 \mathrm{D}$ mesh can be seen as the parameter domain of some parameterization-based remeshing techniques such as the well-known geometry images [14].

To get a 2D semiregular mesh, we adopt a coarse-to-fine approach. We first create a base mesh that is a very coarse mesh of the POI region, using the three following steps, depicted in Figures 3(a) and 3(b):

1. Four points are initialized to the corners of the stereo image;

2. A GPU-parallelized k-nearest neighbors $(\mathrm{kNN})$ technique displaces these points to their respective nearest pixels in the POI region (arrows in Figure 3(b));

3. The connectivity of the two coarse triangles is created.

The connectivity of finer resolutions is generated by midpoint subdivisions: each edge is split into two smaller edges by adding a vertex at its middle point, and each triangle is split into four smaller triangles. Such a mesh is called semiregular because the added vertices are always regular (valency 6, i.e. they are connected to 6 neighbor vertices). The middle point of each edge does not necessary belong to the POI region, therefore the newly added vertex is moved to the closest pixel in the POI region according to the $\mathrm{kNN}$ technique. Figure 3 (c) shows the resulting $2 \mathrm{D}$ semiregular mesh. The same procedure can be done several times to get a more dense semiregular mesh: Figure 3(d) depicts the third level of connectivity defined by $2 \times 4 \times 4$ triangles. 


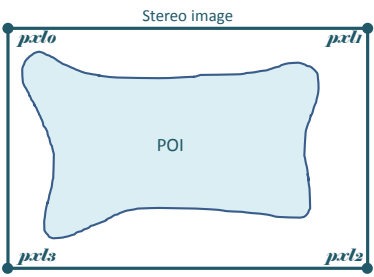

(a)

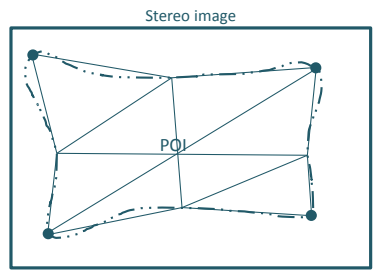

(c)

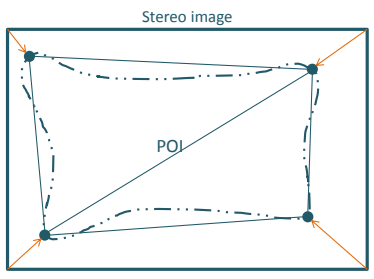

(b)

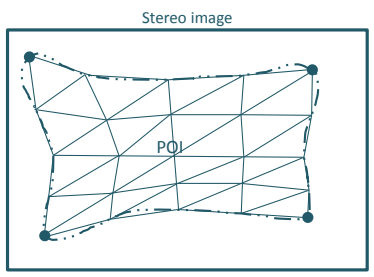

(d)
Fig. 3. Coarse-to-fine generation of the 2D semiregular mesh. (a) Initialisation step: four vertices are initialized to the corners of the stereo image; (b) creation of the base mesh; (c) and (d) the two first resolutions obtained with a midpoint subdivision and displacement of the added vertices to the closest pixels in the POI region.

\section{Computation of the $3 \mathrm{D}$ coordinates}

To retrieve the final 3D semiregular mesh, the 3D coordinates of each point of the 2D semiregular mesh have to be computed. We use the same technique than the classical procedure, described in Figure 1(a). The corresponding 3D coordinates are computed with the classical triangulation technique [13], by dint of the calibration parameters of the scanner.

By iterating this technique over each vertex of the $2 \mathrm{D}$ semiregular mesh, we create the final 3D semiregular mesh, as shown in Figure 2. We observe that the resulting semiregular mesh approximates finely the original surface at different levels of resolution, while avoiding the generation of a dense mesh: the entire point cloud contains 93,595 3D points, while our finest resolution mesh is only composed of 4,225 vertices. Moreover, our reconstruction scheme is fast and easy to implement.

\section{DENTS DETECTION ON SMOOTH SURFACES}

We now present the second part of our algorithm, i.e. the technique that enables to detect automatically some local deformations on a smooth surface defined by a semiregular mesh $M_{s r}$ ( $p$ levels of details). As our algorithm is based on MRA, we first recall few notions about it.

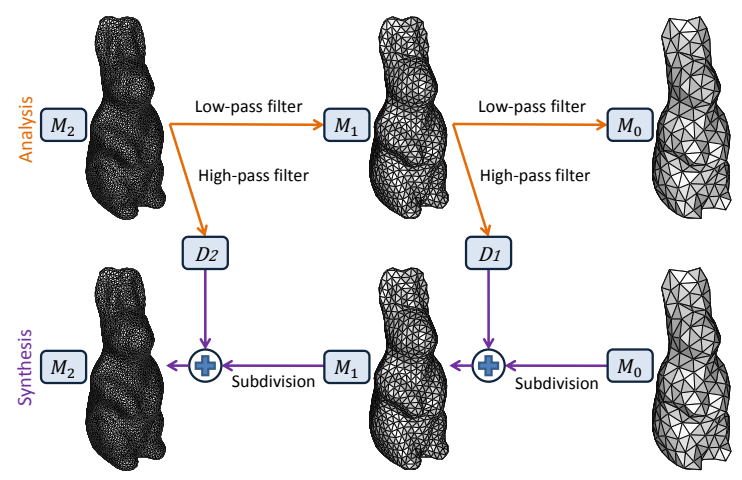

Fig. 4. Multiresolution analysis (MRA) and synthesis on RABBIT.

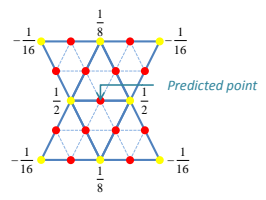

Fig. 5. Mask of the butterfly subdivision scheme.

\subsection{Principle of a multiresolution analysis (MRA)}

MRA consists in decomposing a mesh $M_{s r}=M_{p}$ into a low frequency mesh $M_{0}$ and $p$ subbands of wavelet coefficients. It can be done by applying $p$ times a wavelet transform. Let us denote $\mathbf{V}_{j}$ the set of vertices of a given mesh $M_{j}$ at resolution $j$. Applying a wavelet transform to $M_{j}$ gives the lower resolution mesh $M_{j-1}$ (low-pass filtering) defined by a set of vertices $\mathbf{V}_{j-1}$, and a subband of $3 \mathrm{D}$ wavelet coefficients $\mathbf{D}_{j}$ (high-pass filtering): see Figure 4. Such analysis can be done with a butterfly-based lifting scheme [15]:

- Split. The vertices $\mathbf{V}_{j}$ are split into two subsets $\mathbf{V}_{j}^{0}$ and $\mathbf{V}_{j}^{1}$ (yellow and red points in Figure 5, respectively);

- Predict. We take as input the subset $\mathbf{V}_{j}^{0}$ and predict the positions of the vertices $\mathbf{V}_{j}^{1}$ by using the butterfly subdivision scheme, whose mask is depicted in Figure 5 [16], so that $\mathbf{V}_{j}^{1}=P\left(\mathbf{V}_{j}^{0}\right)$ (i.e. the center of the mask corresponds to the vertex for which we want to predict the position). The prediction errors are the wavelet coefficients $\mathbf{D}_{j}$;

- Update. (Optional) We take as input $\mathbf{D}_{j}$ and modify the positions of the vertices $\mathbf{V}_{j}^{0}$ using an update operator $U$. We finally obtain the low resolution mesh $M_{j-1}$, defined by a set of vertices $\mathbf{V}_{j-1}$.

The synthesis is the opposite operation. For a given resolution $j$, the butterfly scheme is applied to $M_{j-1}$ to get the predicted mesh $M_{j}^{\prime}$, and its difference with $M_{j}$ is compensated by adding the wavelet coefficients $\mathbf{D}_{j}$ (see Figure 4 , above). 


\subsection{Proposed method}

Our detection algorithm is presented in Figure 6.

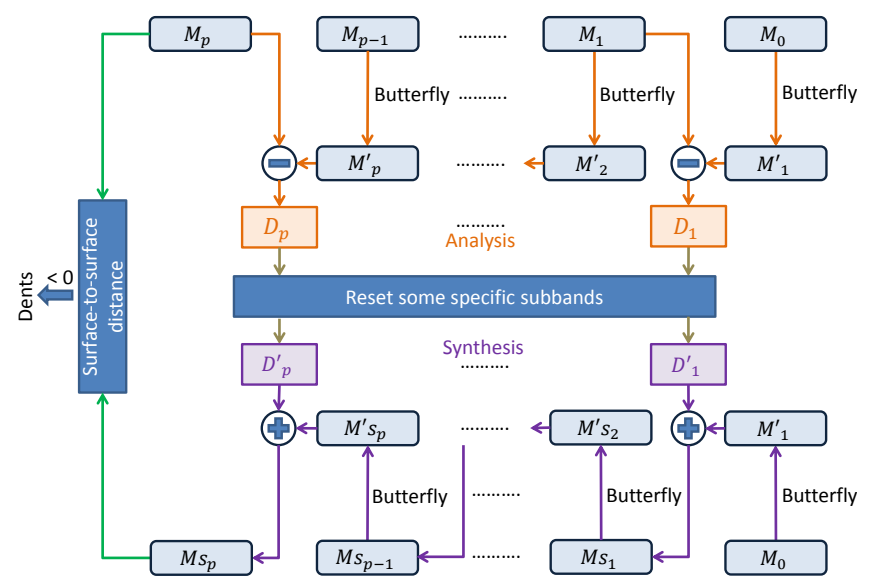

Fig. 6. Detection algorithm.

The first step consists in applying the MRA based on the butterfly-based lifting scheme to $M_{s r}=M_{p}$ (without update step). We obtain the $p$ subbands of coefficients and the low frequency mesh $M_{0}$. Then, several intermediate subbands of wavelet coefficients are reset (all the coefficients are set to zero). Afterwards, the synthesis is done, to obtain the semiregular mesh $M s_{p}$. As some subbands have been reset and the butterfly scheme is interpolating, the mesh $M_{s m}=M s_{p}$ is a smooth version of the original mesh $M_{s r}$. The geometric differences between $M_{s r}$ and $M_{s m}$ thus represent the local deformations. Therefore, signed distances between these two meshes are finally computed: the negative distances highlight the dents (convex parts of $M_{s r}$ ). Concerning the reset of the intermediate subbands, we remarked that 8 levels of details are sufficient to recover all artifacts. More precisely, the first and the last subbands $\mathbf{D}_{1}$ and $\mathbf{D}_{p}$ are never reset, since the latter contains mainly noise, and the first is required to acquire the general shape of the scanned surface. For instance, as the coarse mesh $M_{0}$ of a curved surface is flat (it only contains 2 triangles), the first subband of details $\mathbf{D}_{1}$ is necessary to ensure a correct approximation of the surface. During our experimentations, we noticed that resetting the subbands from the fourth resolution is efficient for detecting dents on curved surfaces, whereas it is more adapted to start the reset phase from the third resolution for flat surfaces.

\section{EXPERIMENTAL RESULTS}

In this section, we present several results to show the efficiency of our method, on scanned surfaces. Figure 7 presents two cases of semiregular reconstruction and automatic detection. The first example is a plane surface that contains three dents (top). The third to the sixth subbands are automatically reset by our algorithm, and this condition permits to well de- tect the three dents. Second, we apply our method on a more curved surface. Our semiregular reconstruction is still correct, and our algorithm detects efficiently the hollow, by resetting only the resolutions 4 to 6 . These examples show that our algorithm resets, as expected, the subbands from a coarser level in case of flat surfaces than with curved ones.
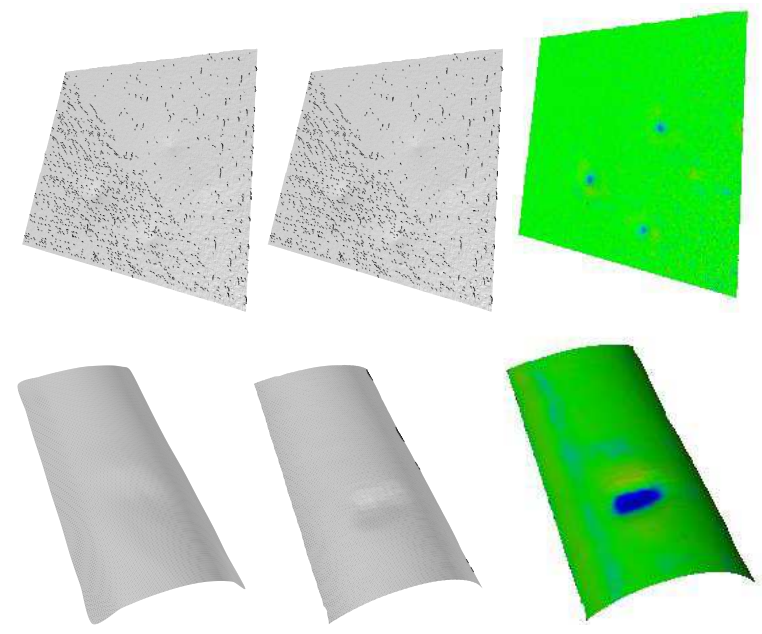

Fig. 7. Results of our multiresolution reconstruction and dents detection on two models: a plane surface that presents three dents, and a curved one with a big dent. Our dents detection algorithm is reliable since every dent has been detected using signed point-to-surface distances.

\section{CONCLUSION}

We presented an algorithm of automatic detection of dents on smooth surfaces especially developed for scanners based on stereovision. The main application is the control of fuselages of airplanes. Our first contribution is an original reconstruction technique from the stereoscopic images, that directly generates a semiregular mesh (unlike the current scanning systems that only generate point clouds). This is an advantage of our method, since the semiregular connectivity is of high interest for many applications in computer graphics. In our case, this semiregular connectivity is used to detect dents on the scanned surfaces, thanks to our algorithm based on the butterfly lifting scheme (second contribution).

These results are only preliminary results, and many promising ways will be explored to improve the two parts of our algorithm. For instance, we are currently working to enforce the robustness of the detection tool, in particular on detecting unexpected dents or bumps when the fuselage also contains local deformations such as rivets or welds. We are also working on the improvement of the quality of the semiregular meshes generated by our direct reconstruction technique, in particular with more complex scanned surfaces. 


\section{REFERENCES}

[1] Peter K. Allen, Alejandro Troccoli, Benjamin Smith, Stephen Murray, Ioannis Stamos, and Marius Leordeanu, "New methods for digital modeling of historic sites," IEEE Comput. Graph. Appl., vol. 23, no. 6, pp. 32-41, Nov. 2003.

[2] Gabriele Guidi, Laura Micoli, Michele Russo, Bernard Frischer, Monica De Simone, Alessandro Spinetti, and Luca Carosso, "3d digitization of a large model of imperial rome," in Proceedings of the Fifth International Conference on 3-D Digital Imaging and Modeling, Washington, DC, USA, 2005, 3DIM '05, pp. 565572, IEEE Computer Society.

[3] Nico Cornelis, Kurt Cornelis, and Luc Van Gool, "Fast compact city modeling for navigation previsualization," in Proceedings of the 2006 IEEE Computer Society Conference on Computer Vision and Pattern Recognition - Volume 2, Washington, DC, USA, 2006, CVPR '06, pp. 1339-1344, IEEE Computer Society.

[4] Nico Cornelis, Bastian Leibe, Kurt Cornelis, and Luc Gool, "3d urban scene modeling integrating recognition and reconstruction," Int. J. Comput. Vision, vol. 78, no. 2-3, pp. 121-141, July 2008.

[5] B. Micusik and J. Kosecka, "Piecewise planar city 3d modeling from street view panoramic sequences," 2013 IEEE Conference on Computer Vision and Pattern Recognition, vol. 0, pp. 2906-2912, 2009.

[6] Yasutaka Furukawa and Jean Ponce, "Accurate, dense, and robust multiview stereopsis," IEEE Transactions on Pattern Analysis and Machine Intelligence, vol. 32, no. 8, pp. 1362-1376, 2010.

[7] M. Jancosek and T. Pajdla, "Multi-view reconstruction preserving weakly-supported surfaces," in Proceedings of the 2011 IEEE Conference on Computer Vision and Pattern Recognition, Washington, DC, USA, 2011, CVPR '11, pp. 3121-3128, IEEE Computer Society.

[8] Tamy Boubekeur, Wolfgang Heidrich, Xavier Granier, and Christophe Schlick, "Volume-surface trees," Computer Graphics Forum (Proceedings of EUROGRAPHICS 2006), vol. 25, no. 3, pp. 399-406, 2006.

[9] John Michael Lounsbery, Multiresolution Analysis for Surfaces of Arbitrary Topological Type, Phd thesis, University of Washington, September 1994.

[10] F. Payan and M. Antonini, "Mean square error approximation for wavelet-based semiregular mesh compression," Transactions on Visualization and Computer Graphics (TVCG), vol. 12, 2006.
[11] Ulf Labsik, Leif Kobbelt, Robert Schneider, and HansPeter Seidel, "Progressive transmission of subdivision surfaces," Computational Geometry, vol. 15, no. 1-3, pp. 25-39, 2000.

[12] Daniel Scharstein and Richard Szeliski, "A taxonomy and evaluation of dense two-frame stereo correspondence algorithms," Int. J. Comput. Vision, vol. 47, no. 1-3, pp. 7-42, Apr. 2002.

[13] R. I. Hartley and A. Zisserman, Multiple View Geometry in Computer Vision, Cambridge University Press, ISBN: 0521540518, second edition, 2004.

[14] Xianfeng Gu, Steven J. Gortler, and Hugues Hoppe, "Geometry images," in Proceedings of ACM SIGGRAPH'02, 2002, vol. 21, pp. 355-361.

[15] Wim Sweldens, "The lifting scheme: A construction of second generation wavelets," SIAM Journal on Mathematical Analysis, vol. 29, no. 2, pp. 511-546, 1998.

[16] Nira Dyn, David Levin, and John A. Gregory, "A butterfly subdivision scheme for surface interpolation with tension control," ACM Transactions on Graphics, vol. 9, no. 2, pp. 160-169, 1990. 